\title{
Some applications of differential subordinations in the geometric function theory
}

\author{
Janusz Sokół* \\ Dedicated to Professor Hari M Srivastava
}

"Correspondence: jsokol@prz.edu.pl Department of Mathematics, Rzeszów University of Technology, al. Powstańców Warszawy 12, Rzeszów, 35-959, Poland

\begin{abstract}
Let $\mathcal{A}$ denote the class of functions $f$, which are analytic in the open unit disc and normalized by $f(0)=f^{\prime}(0)-1=0$. We investigate the connection of the quantity $z\left(f^{\prime}(z)-1\right) / f(z)$ with $f(z) / z$. Obtained results are the extensions of those presented by Tuneski and Obradović (Comput. Math. Appl. 62:3438-3445, 2011). Moreover, we solve the open problems posed in the paper above.

MSC: Primary 30C45; secondary 30C80

Keywords: differential subordination; starlike function; convex function; order of starlikeness; order of convexity; best dominant
\end{abstract}

\section{Introduction}

Let $\mathcal{A}(n), n \in \mathbb{N}$, denote the class of functions of the form

$$
f(z)=z+a_{n+1} z^{n+1}+a_{n+2} z^{n+2}+\cdots,
$$

which are analytic in the open unit disc $\mathbb{U}=\{z:|z|<1\}$ on the complex plane $\mathbb{C}$. For two analytic functions $f, g$, we say that $f$ is subordinate to $g$, written as $f \prec g$, if and only if there exists an analytic function $\omega$ with property $|\omega(z)| \leq|z|$ in $\mathbb{U}$ such that $f(z)=g(\omega(z))$. In particular, if $g$ is univalent in $\mathbb{U}$, then we have the following equivalence

$$
f(z) \prec g(z) \quad \Leftrightarrow \quad f(0)=g(0) \quad \text { and } \quad f(\mathbb{U}) \subset g(\mathbb{U}) .
$$

The idea of subordination was used for defining many of classes of functions studied in the geometric function theory. For obtaining the main result, we shall use the method of differential subordinations. The main result in the theory of differential subordinations was introduced by Miller and Mocanu in [1,2]. A function $p$, analytic in $\mathbb{U}$, is said to satisfy a first order differential subordination if

$$
\phi\left(p(z), z p^{\prime}(z)\right) \prec h(z)
$$

where $\left(p(z), z p^{\prime}(z)\right) \in D \subset \mathbb{C}^{2}, \phi: \mathbb{C}^{2} \rightarrow \mathbb{C}$ is analytic in $D, h$ is analytic and univalent in $\mathbb{U}$. The function $q$ is said to be a dominant of the differential subordination (1.2) if $p \prec q$ for

\section{Springer}

(c) 2013 Sokół; licensee Springer. This is an Open Access article distributed under the terms of the Creative Commons Attribution License (http://creativecommons.org/licenses/by/2.0), which permits unrestricted use, distribution, and reproduction in any medium, provided the original work is properly cited. 
all $p$ satisfying (1.2). If $\widetilde{q}$ is a dominant of (1.2) and $\widetilde{q} \prec q$ for all dominants $q$ of (1.2), then we say that $\widetilde{q}$ is the best dominant of the differential subordination (1.2).

By using a Miller-Mocanu lemma on the first order differential subordination, the authors of [3] proved the following theorem.

Theorem 1.1 [3] Let $f \in \mathcal{A}(1), f(z) / z \neq 0$ for all $z \in \mathbb{U}$ and $0<\mu \leq 1$. If

$$
\frac{z\left(f^{\prime}(z)-1\right)}{f(z)} \prec \frac{2 \mu z}{1+\mu z} \equiv h_{1}(z) \quad(z \in \mathbb{U})
$$

then

$$
\frac{f(z)}{z}-1 \prec \mu z
$$

and $\mu z$ is the best dominant of (1.4). Furthermore,

$$
\left|\frac{f(z)}{z}-1\right|<\mu \quad(z \in \mathbb{U})
$$

and this conclusion is sharp, i.e., in inequality (1.5), $\mu$ cannot be replaced by a smaller number such that the implications holds.

\section{Main results}

It is easy to verify that under the assumptions of Theorem 1.1, the function $p(z)=z / f(z)$ is analytic in $\mathbb{U}$, and that the subordination (1.3) becomes

$$
\left[-\left(p(z)+\frac{z p^{\prime}(z)}{p(z)}\right)+1\right] \prec h_{1}(z)=\frac{2 \mu z}{1+\mu z} \quad(z \in \mathbb{U}) .
$$

Thus, (1.3) can be rewritten as the Briot-Bouquet-type differential subordination

$$
s(z)+\frac{z s^{\prime}(z)}{\beta s(z)+\gamma} \prec h(z) \quad(z \in \mathbb{U})
$$

with $s(z)=p(z), \beta=1, \gamma=0, h(z)=1-h_{1}(z)$.

One of the basic results in the theory of Briot-Bouquet differential subordinations is a theorem from [4], which says (in its particular case) that if $h$ is convex univalent with positive real part in $\mathbb{U}$ and the functions $s, h$ satisfy (2.1), then $s \prec h$. It can sometimes be improved if we know more about the function $h$. A better subordination will be derived by applying the results from the book [2]. After some adaptation, Theorem 3.2j [2, p.97] becomes the following lemma.

Lemma 2.1 [2] Suppose that $\beta>0$ and $n$ is a positive integer. Let us denote

$$
\mathcal{R}_{\beta, n}(z)=\beta \frac{1+z}{1-z}+\frac{2 n z}{1-z^{2}} \quad(z \in \mathbb{U})
$$

and let $h$ be a convex univalent function in $\mathbb{U}$ with $h(0)=1$ such that

$$
\beta h(z) \prec \mathcal{R}_{\beta, n}(z) \quad(z \in \mathbb{U}) .
$$


Then the Briot-Bouquet differential equation

$$
q(z)+\frac{n z q^{\prime}(z)}{\beta q(z)}=h(z) \quad(z \in \mathbb{U})
$$

has a univalent solution $q_{n}$ analytic in $\mathbb{U}$. Furthermore, if the functions $h$ and $s(z)=1+$ $a_{n} z^{n}+a_{n+1} z^{n+1}+\cdots$ satisfy the Briot-Bouquet differential subordination

$$
s(z)+\frac{z s^{\prime}(z)}{\beta s(z)} \prec h(z) \quad(z \in \mathbb{U})
$$

then

$$
s(z) \prec q_{n}(z) \prec h(z) \quad(z \in \mathbb{U}),
$$

and the function $q_{n}$ is the best dominant of the subordination (2.4) in the sense that if there exists a function $p$ such that $s(z) \prec p(z)$, then $q_{n}(z) \prec p(z)$.

Notice that the function $\mathcal{R}_{\beta, n}$ is called the open door function, and it is univalent in $\mathbb{U}$, $\mathcal{R}_{\beta, n}(0)=\beta$. Thus, by (1.1), in order to verify (2.3), it is sufficient to show that $h(0)=1$ and $\beta h(\mathbb{U}) \subset \mathcal{R}_{\beta, n}(\mathbb{U})$. The set $\mathcal{R}_{\beta, n}(\mathbb{U})$ is the complex plane with slits along the half-lines $\mathfrak{R e} w=0$ and $|\operatorname{Im} w| \geq \gamma=n \sqrt{1+2 \beta / n}$. For $\mathcal{R}_{4,1}$, these slits are placed in Figure 1 with respect to the circle centered at $w=1$ and the radius $\sqrt{10}$.

If $h$ is a special bilinear transformation

$$
h(z)=\frac{1+A z}{1+B z}, \quad A \in \mathbb{C}, B \in[-1,0], A \neq B,
$$

and if we replace condition (2.3) by a simpler condition $\mathfrak{R e}\{h(z)\}>0$, then it will be satisfied (see [2, p.108]) if and only if

$$
\mathfrak{R e}[\beta(1+A B)] \geq|\beta A+\bar{\beta} B|
$$

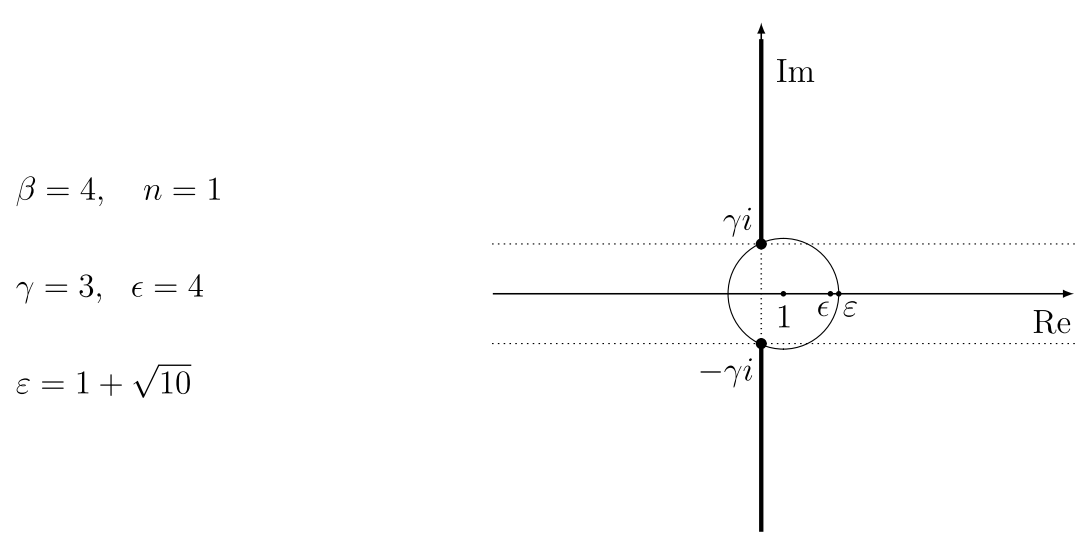

Figure $1 \mathcal{R}_{\beta, n}(\mathbb{U})$. 
when $B \in(-1,0]$, or

$$
\mathfrak{R e} \beta(1+A)>0 \text { and } \mathfrak{R e} \beta(1-A) \geq 0,
$$

when $B=-1$. Then the univalent solution $q_{n}$ of the Briot-Bouquet differential equation

$$
q(z)+\frac{n z q^{\prime}(z)}{\beta q(z)}=\frac{1+A z}{1+B z} \quad(z \in \mathbb{U})
$$

has the form

$$
q_{n}(z)=\frac{1}{\beta g_{n}(z)},
$$

where

$$
g_{n}(z)= \begin{cases}\frac{1}{n} \int_{0}^{1}\left[\frac{1+B t z}{1+B z}\right]^{\frac{\beta}{n}\left(\frac{A}{B}-1\right)} t^{\frac{\beta}{n}-1} \mathrm{~d} t, & \text { if } B \neq 0 \\ \frac{1}{n} \int_{0}^{1} e^{\frac{\beta A(t-1) z}{n}} t^{\frac{\beta}{n}-1} \mathrm{~d} t, & \text { if } B=0 .\end{cases}
$$

Theorem 2.2 Let $f \in \mathcal{A}(n), f(z) / z \neq 0$ for all $z \in \mathbb{U}$. If $f(z) / z=1+a_{n} z^{n}+a_{n+1} z^{n+1}+\cdots$ satisfies the Briot-Bouquet differential subordination

$$
\frac{z\left(f^{\prime}(z)-1\right)}{f(z)} \prec h(z) \quad(z \in \mathbb{U}),
$$

with a convex univalent function $h$, such that $h(0)=0$ and

$$
1-h(z) \prec \mathcal{R}_{1, n}(z) \quad(z \in \mathbb{U}),
$$

where $\mathcal{R}_{1, n}$ is the open door function given in (2.2), then

$$
\frac{f(z)}{z} \prec q_{n}(z) \quad(z \in \mathbb{U})
$$

where

$$
q_{n}(z)=\frac{1}{n} \int_{0}^{1}\left[\frac{H(t z)}{H(z)}\right]^{1 / n} t^{-1} \mathrm{~d} t \quad(z \in \mathbb{U})
$$

and

$$
H(z)=z \exp \int_{0}^{z}-h(t) / t \mathrm{~d} t
$$

and $q_{n}$ is the best dominant of (2.10).

Proof The function $p(z)=z / f(z)=1-a_{n} z^{n}+\cdots$ is analytic in $\mathbb{U}$ and

$$
\frac{z\left(f^{\prime}(z)-1\right)}{f(z)}=-\left(p(z)+\frac{z p^{\prime}(z)}{p(z)}\right)+1 \quad(z \in \mathbb{U}),
$$


hence subordination (2.9) becomes

$$
p(z)+\frac{z p^{\prime}(z)}{p(z)} \prec 1-h(z) \quad(z \in \mathbb{U}) .
$$

By Lemma 2.1, the equation

$$
q(z)+\frac{n z q^{\prime}(z)}{q(z)}=1-h(z) \quad(z \in \mathbb{U})
$$

has the univalent solution of the form $\left\{q_{n}(z)\right\}^{-1}$, where $q_{n}$ is in (2.11) (for more details see $\left[2\right.$, p.86]). Therefore, again by Lemma 2.1, the function $\left\{q_{n}(z)\right\}^{-1}$ is the best dominant of the subordination

$$
p(z)=\frac{z}{f(z)} \prec\left\{q_{n}(z)\right\}^{-1} \quad(z \in \mathcal{U}),
$$

which is equivalent to (2.10) with the best dominant $q_{n}$.

Theorem 2.3 Let $f \in \mathcal{A}(n), f(z) / z \neq 0$ for all $z \in \mathbb{U}$. Assume that $A \in \mathbb{C}, B \in[-1,0], A \neq B$ and that $A$ and $B$ satisfy either (2.5) or (2.6) with $\beta=1$. If $f(z) / z=1+a_{n} z^{n}+a_{n+1} z^{n+1}+\cdots$ satisfies the Briot-Bouquet differential subordination

$$
\frac{z\left(f^{\prime}(z)-1\right)}{f(z)} \prec \frac{(B-A) z}{1+B z} \quad(z \in \mathbb{U})
$$

then

$$
\frac{f(z)}{z} \prec g_{n}(z)= \begin{cases}\frac{1}{n} \int_{0}^{1}\left[\frac{1+B t z}{1+B z}\right]^{\frac{1}{n}\left(\frac{A}{B}-1\right)} t^{\frac{1}{n}-1} \mathrm{~d} t, & \text { if } B \neq 0, \\ \frac{1}{n} \int_{0}^{1} e^{\frac{A(t-1) z}{n}} t^{\frac{1}{n}-1} \mathrm{~d} t, & \text { if } B=0,\end{cases}
$$

and $g_{n}(z)$ is the best dominant of (2.14).

Proof Subordination (2.13) with $p(z)=z / f(z)=1-a_{n} z^{n}+\cdots$ becomes

$$
p(z)+\frac{z p^{\prime}(z)}{p(z)}=\frac{1+A z}{1+B z} \quad(z \in \mathbb{U})
$$

If we return to Lemma 2.1 with $\beta=1$ and to the remarks below Figure 1, then we can see that under assumptions (2.5) or (2.6) there exists a univalent solution $q_{n}$ of equation (2.7). Moreover, this function $q_{n}$ has the form (2.8) and is best dominant of the subordination $p \prec q_{n}$. It also implies $1 / p \prec g_{n}$, which is equivalent to (2.14).

Theorem 2.3 with $n=1, A=-\mu$ and $B=\mu$ becomes the earlier Theorem 1.1, cited in the first section. Notice that for $B \in(-1,0]$ the function on the right hand side of (2.13) maps the open unit disc $\mathbb{U}$ onto the disc

$$
D(C, R)=\{w \in \mathbb{C}:|w-C|<R\},
$$


where

$$
C=\frac{B(A-B)}{1-B^{2}}, \quad R=\frac{|A-B|}{1-B^{2}} .
$$

This function is also univalent in $\mathbb{U}$ whence, by (1.1), Theorem 2.3 can be written in the following form.

Corollary 2.4 Let $f \in \mathcal{A}(n), f(z) / z \neq 0$ for all $z \in \mathbb{U}$. If $f(z) / z=1+a_{n} z^{n}+a_{n+1} z^{n+1}+\cdots$ satisfies the inequality

$$
\left|\frac{z\left(f^{\prime}(z)-1\right)}{f(z)}-\frac{B(A-B)}{1-B^{2}}\right|<\frac{|A-B|}{1-B^{2}} \quad(z \in \mathbb{U})
$$

where $A \in \mathbb{C}, B \in(-1,0], A \neq B$ satisfy (2.5) with $\beta=1$, then

$$
\frac{f(z)}{z} \prec g_{n}(z)=\left\{\begin{array}{ll}
\frac{1}{n} \int_{0}^{1}\left[\frac{1+B t z}{1+B z}\right]^{\frac{1}{n}\left(\frac{A}{B}-1\right)} t^{\frac{1}{n}-1} \mathrm{~d} t, & \text { if } B \neq 0, \\
\frac{1}{n} \int_{0}^{1} e^{\frac{A(t-1) z}{n}} t^{\frac{1}{n}-1} \mathrm{~d} t, & \text { if } B=0
\end{array} \quad(z \in \mathbb{U}),\right.
$$

and it is the best dominant of (2.15). Moreover, if $A$ satisfies (2.6) with $\beta=1$, and iff $f(z) / z=$ $1+a_{n} z^{n}+a_{n+1} z^{n+1}+\cdots$ satisfies condition (2.13) with $B=-1$, i.e.,

$$
\mathfrak{R e}\left\{\frac{z\left(f^{\prime}(z)-1\right)}{(1+A) f(z)}\right\}<\frac{1}{2}, \quad(z \in \mathbb{U}),
$$

then

$$
\frac{f(z)}{z} \prec g_{n}(z)=\frac{1}{n} \int_{0}^{1}\left[\frac{1-z}{1-t z}\right]^{\frac{1+A}{n}} t^{\frac{1}{n}-1} \mathrm{~d} t, \quad(z \in \mathbb{U}),
$$

and $g_{n}(z)$ is the best dominant of (2.16).

If $B=0, A=\lambda \in(0,1], n=1$, then (2.5) is satisfied, and Corollary 2.4 becomes the following one, which is a generalization of Corollary 1 in [3].

Corollary 2.5 Let $\lambda \in(0,1], f \in \mathcal{A}(1)$. Suppose that $f(z) / z \neq 0$ for all $z \in \mathbb{U}$. Iff satisfies the inequality

$$
\left|\frac{z\left(f^{\prime}(z)-1\right)}{f(z)}\right|<\lambda \quad(z \in \mathbb{U}),
$$

then

$$
\frac{f(z)}{z}-1 \prec \frac{e^{\lambda z}-1}{\lambda z e^{\lambda z}}-1=\sum_{k=1}^{\infty} \frac{(-1)^{k}(\lambda z)^{k}}{(k+1) !} \quad(z \in \mathbb{U}),
$$

and it is the best dominant of (2.18).

Note that instead of (2.18), in [3] is the inequality

$$
\left|\frac{f(z)}{z}-1\right|<\mu \quad(z \in \mathbb{U}),
$$


where $\mu=\frac{\lambda}{2-\lambda}$, under the same assumption as in Corollary 2.5. In [3], the authors posed also the problem of finding the smallest number $\mu$ such that (2.19) holds under the assumptions of Corollary 2.5. In view of (2.18), solving this problem is equivalent to finding

$$
\max \left\{\left|\frac{e^{\lambda z}-1}{\lambda z e^{\lambda z}}-1\right|: z \in \mathcal{U}\right\}
$$

To find (2.20), we use the Taylor series (2.18) with $z=e^{i t}$.

$$
\begin{aligned}
\left|\frac{e^{\lambda z}-1}{\lambda z e^{\lambda z}}-1\right| & \leq \sum_{k=1}^{\infty}\left|\frac{(-1)^{k+1} \lambda^{k} e^{k i t}}{(k+1) !}\right| \\
& \leq \sum_{k=1}^{\infty} \frac{\lambda^{k}}{(k+1) !} \\
& =\frac{1}{\lambda} \sum_{k=1}^{\infty} \frac{\lambda^{k+1}}{(k+1) !} \\
& =\frac{1}{\lambda}\left(e^{\lambda}-1-\lambda\right) .
\end{aligned}
$$

Moreover,

$$
\left.\left|\frac{e^{\lambda z}-1}{\lambda z e^{\lambda z}-1}\right|\right|_{z=-1}=\frac{1}{\lambda}\left(e^{\lambda}-1-\lambda\right)
$$

hence

$$
\max \left\{\left|\frac{e^{\lambda z}-1}{\lambda z e^{\lambda z}}-1\right|: z \in \mathcal{U}\right\}=\frac{1}{\lambda}\left(e^{\lambda}-1-\lambda\right)
$$

For all $\lambda \in(0,1]$, the number (2.21) is better than the bound in (2.19), because it is smaller than $\mu=\frac{\lambda}{2-\lambda}$, given in [3]. This is because

$$
\begin{aligned}
\frac{\lambda}{2-\lambda} & =\frac{1}{\lambda} \frac{\lambda^{2} / 2}{1-\lambda / 2} \\
& =\frac{1}{\lambda} \sum_{k=1}^{\infty} \frac{\lambda^{k+1}}{2^{k}} \\
& \geq \frac{1}{\lambda} \sum_{k=1}^{\infty} \frac{\lambda^{k+1}}{(k+1) !} \\
& =\frac{1}{\lambda}\left\{-1-\lambda+\sum_{k=0}^{\infty} \frac{\lambda^{k}}{k !}\right\} \\
& =\frac{1}{\lambda}\left(e^{\lambda}-1-\lambda\right) .
\end{aligned}
$$

Therefore, the following corollary contains the solution of the first open problem posed in [3]. 
Corollary 2.6 Let $\lambda \in(0,1], f \in \mathcal{A}(1)$. Suppose that $f(z) / z \neq 0$ for all $z \in \mathbb{U}$. If $f$ satisfies the inequality

$$
\left|\frac{z\left(f^{\prime}(z)-1\right)}{f(z)}\right|<\lambda \quad(z \in \mathbb{U})
$$

then

$$
\left|\frac{f(z)}{z}-1\right|<\frac{e^{\lambda}-1-\lambda}{\lambda} \quad(z \in \mathbb{U})
$$

and this bound is the best possible.

The second open problem posed in [3] is to find the sharp version of the following corollary.

Corollary 2.7 [3] Let $\lambda \in(0,1], f \in \mathcal{A}(1)$. Iff satisfies the inequality

$$
\left|f^{\prime}(z)-1\right|<\lambda\left|\frac{f(z)}{z}\right| \quad(z \in \mathbb{U})
$$

then

$$
\left|f^{\prime}(z)-1\right|<\frac{2 \lambda}{2-\lambda} \quad(z \in \mathbb{U})
$$

and

$$
\mathfrak{R e}\left\{\frac{z f^{\prime}(z)}{f(z)}\right\}>1-\frac{3 \lambda}{2} \quad(z \in \mathbb{U}) .
$$

Using the earlier results, we can improve the corollary above.

Corollary 2.8 Under the assumptions of Corollary 2.7, we have

$$
\left|f^{\prime}(z)-1\right|<e^{\lambda}-1 \quad(z \in \mathbb{U})
$$

and

$$
\mathfrak{R e} \frac{z f^{\prime}(z)}{f(z)}>\frac{\lambda\left(2-e^{\lambda}\right)}{e^{\lambda}-1} \quad(z \in \mathbb{U})
$$

Proof Inequality (2.22) follows that $f(z) / z \neq 0$ for all $z \in \mathbb{U}$. Hence (2.22) is the same as (2.17), thus, from (2.18), we obtain

$$
\begin{aligned}
\frac{f(z)}{z} & \prec \frac{e^{\lambda z}-1}{\lambda z e^{\lambda z}} \\
& =1+\sum_{k=1}^{\infty} \frac{(-1)^{k+1}(\lambda z)^{k}}{(k+1) !} \quad(z \in \mathcal{U}) .
\end{aligned}
$$


Therefore, for $z=e^{i t}$, we obtain

$$
\begin{aligned}
\left|\frac{f(z)}{z}\right| & \leq\left|\frac{e^{\lambda z}-1}{\lambda z e^{\lambda z}}\right| \\
& \leq 1+\sum_{k=1}^{\infty}\left|\frac{(-1)^{k+1} \lambda^{k} e^{k i t}}{(k+1) !}\right| \\
& \leq 1+\sum_{k=1}^{\infty} \frac{\lambda^{k}}{(k+1) !} \\
& =\frac{1}{\lambda}\left\{\lambda+\sum_{k=1}^{\infty} \frac{\lambda^{k+1}}{(k+1) !}\right\} \\
& =\frac{1}{\lambda}\left(e^{\lambda}-1\right) .
\end{aligned}
$$

Moreover,

$$
\left.\left|\frac{e^{\lambda z}-1}{\lambda z e^{\lambda z}}\right|\right|_{z=-1}=\frac{1}{\lambda}\left(e^{\lambda}-1\right)
$$

So the bound (2.27) is the best possible. Applying (2.27) in (2.22), we directly obtain (2.25). Making use of (2.22) and of (2.27), we get the inequality

$$
\begin{aligned}
\mathfrak{R e} \frac{z f^{\prime}(z)}{f(z)} & =\mathfrak{R e} \frac{z\left(f^{\prime}(z)-1\right)}{f(z)}+\mathfrak{R e} \frac{z}{f(z)}>-\lambda+\frac{\lambda}{e^{\lambda}-1} \\
& =\frac{\lambda\left(2-e^{\lambda}\right)}{e^{\lambda}-1} \quad(z \in \mathbb{U}),
\end{aligned}
$$

which is (2.26).

For all $\lambda \in(0,1]$ the bound (2.25) is smaller than the bound $\frac{2 \lambda}{2-\lambda}$, given in (2.24). To show this, observe that

$$
\frac{2 \lambda}{2-\lambda}=\frac{\lambda}{1-\lambda / 2}=\sum_{k=1}^{\infty} \frac{\lambda^{k}}{2^{k-1}}>\sum_{k=1}^{\infty} \frac{\lambda^{k}}{k !}=-1+\sum_{k=0}^{\infty} \frac{\lambda^{k}}{k !}=e^{\lambda}-1
$$

Similarly, for all $\lambda \in(0,1]$, the bound (2.26) is greater than the bound $1-3 \lambda / 2$, given in (2.23). To show this, observe that the inequality

$$
\frac{\lambda\left(2-e^{\lambda}\right)}{e^{\lambda}-1}>1-\frac{3 \lambda}{2}
$$

that has to be proved, after some calculations, becomes

$$
\frac{2 \lambda}{2-\lambda}>e^{\lambda}-1
$$

which was proved above in (2.28). The function $f(z)=\left(e^{\lambda z}-1\right) / \lambda$ shows that bound (2.25) delivers the sharp version of (2.23), so this is the solution of the first part of the second 
open problem posed in [3]. The bound (2.26) does not seem to be the best possible. We conjecture that the best possible bound is

$$
\mathfrak{R e} \frac{z f^{\prime}(z)}{f(z)}>\frac{\lambda}{e^{\lambda}-1} \quad(z \in \mathbb{U}),
$$

which is suggested by the function $f(z)=\left(e^{\lambda z}-1\right) / \lambda$.

In [5], Robertson introduced the classes $\mathcal{S}_{\alpha}^{*}, \mathcal{K}_{\alpha}$ of starlike and convex functions of order $\alpha \leq 1$, which are defined by

$$
\begin{aligned}
& \mathcal{S}_{\alpha}^{*}:=\left\{f \in \mathcal{A}: \mathfrak{R e} \frac{z f^{\prime}(z)}{f(z)}>\alpha, z \in \mathbb{D}\right\} \\
& \mathcal{K}_{\alpha}:=\left\{f \in \mathcal{A}: \mathfrak{R e}\left(1+\frac{z f^{\prime \prime}(z)}{f^{\prime}(z)}\right)>\alpha, z \in \mathbb{D}\right\}=\left\{f \in \mathcal{A}: z f^{\prime}(z) \in \mathcal{S}_{\alpha}^{*}\right\} .
\end{aligned}
$$

If $\alpha \in[0 ; 1)$, then a function in each of these sets is univalent, if $\alpha<0$, it may fail to be univalent. In particular, we have $\mathcal{S}_{0}^{*}=\mathcal{S}^{*}, \mathcal{K}_{0}=\mathcal{K}$, the usual classes of starlike and of convex functions, respectively. In Corollary 2.8, we obtained the order of starlikeness for functions satisfying (2.22), so we have

$$
\left|f^{\prime}(z)-1\right|<\lambda\left|\frac{f(z)}{z}\right| \Rightarrow f \in \mathcal{S}_{\alpha}^{*}
$$

where

$$
\alpha=\frac{\lambda\left(2-e^{\lambda}\right)}{e^{\lambda}-1}, \quad \lambda \in(0,1]
$$

Therefore, if $\lambda \in(0, \log 2]$, then condition (2.22) is sufficient for $f$ to be starlike, namely,

$$
\left|f^{\prime}(z)-1\right|<\lambda\left|\frac{f(z)}{z}\right| \Rightarrow f \in \mathcal{S}^{*} .
$$

Notice that the number $\log 2 \approx 0.693$ is better than $2 / 3$ given in this place in [3]. Recall that in (2.29), we conjectured that for all $\lambda \in(0,1]$ a function satisfying (2.22) is starlike, even more, starlike of order $\lambda /\left(e^{\lambda}-1\right)$. Using $z f^{\prime}(z)$ instead of $f$ in Corollary 2.8, we obtain the order of convexity for a function satisfying (2.22), so we have

$$
\left|z f^{\prime \prime}(z)+f^{\prime}(z)-1\right|<\lambda\left|f^{\prime}(z)\right| \Rightarrow\left(1+\frac{z f^{\prime \prime}(z)}{f^{\prime}(z)}\right)>\alpha
$$

where $\alpha$ is described in (2.30), which means that $f \in \mathcal{K}_{\alpha}$. For some similar conditions for starlikeness of order $\alpha$, we also refer to $[6,7]$ and to [8]. 
References

1. Miller, SS, Mocanu, PT: Differential subordinations and univalent functions. Mich. Math. J. 28, 157-171 (1981)

2. Miller, SS, Mocanu, PT: Differential Subordinations: Theory and Applications. Series of Monographs and Textbooks in Pure and Applied Mathematics, vol. 225. Dekker, New York (2000)

3. Tuneski, N, Obradović, M: Some properties of certain expressions of analytic functions. Comput. Math. Appl. 62 3438-3445 (2011)

4. Eenigenburg, P, Miller, SS, Mocanu, PT, Reade, MO: General Inequalities 3. International Series of Numerical Mathematics, vol. 64, pp. 339-348. Birkhäuser, Basel (1983)

5. Robertson, MS: On the theory of univalent functions. Ann. Math. 37, 374-408 (1936)

6. Nunokawa, M, Kuroki, K, Sokół, J, Owa, S: New extensions concerned with results by Ponnusamy and Karunakaran. Adv. Differ. Equ. 2013, 134 (2013)

7. Sokół, J: On a condition for starlikeness. J. Math. Anal. Appl. 352, 696-701 (2009)

8. Sokół, J, Nunokawa, M: On some sufficient conditions for univalence and starlikeness. J. Inequal. Appl. 2012, 282 (2012)

doi:10.1186/1029-242X-2013-389

Cite this article as: Sokół: Some applications of differential subordinations in the geometric function theory. Journal of Inequalities and Applications 2013 2013:389.

\section{Submit your manuscript to a SpringerOpen ${ }^{\circ}$ journal and benefit from:}

- Convenient online submission

- Rigorous peer review

- Immediate publication on acceptance

- Open access: articles freely available online

- High visibility within the field

- Retaining the copyright to your article 